\title{
Resultados del drenaje de la vía biliar por CPRE en pacientes con edad geriátrica
}

\author{
J. García-Cano, J. A. González Martín¹, M. J. Morillas Ariño, J. I. Pérez García, E. Redondo Cerezo, \\ C. Jimeno Ayllón, M. Viñuelas Chicano, N. Sánchez Manjavacas, C. J. Gómez Ruiz, M. G. Pérez Vigara \\ y A. Pérez Sola
}

Sección de Aparato Digestivo. Hospital Virgen de la Luz. Cuenca. . Servicio de Gastroenterología. Hospital Ramón y Cajal. Madrid

\section{RESUMEN}

Antecedentes y objetivo: la colangiopancreatografía retrógrada endoscópica (CPRE) es habitualmente la técnica de elección para desobstruir la vía biliar. Una gran parte de los pacientes a los que se realiza esta intervención están en edad geriátrica (mayores de 75 años). Nuestro objetivo ha sido valorar la eficacia de la CPRE en este grupo de pacientes, comparado con los de menor edad.

Pacientes y métodos: estudio retrospectivo en el que se han incluido los pacientes a quienes se realizó terapéutica biliar endoscópica mediante CPRE en un periodo de cuatro años (20022005).

Resultados: se realizó CPRE a 178 pacientes en edad geriátrica y a 159 de menor edad. No hubo diferencias en el éxito del drenaje biliar (97,7-98,7\%), en el número de complicaciones (11,8-14,4\%), ni en la mortalidad (1,1-0,6\%). Por el contrario, en el grupo geriátrico hubo más coledocolitiasis $(57,3-39,6 \%$, $p=$ $0,004)$ y se utilizaron más prótesis metálicas autoexpandibles para drenar la ictericia obstructiva tumoral $(47-8 \%, p=0,0035)$. En el grupo de menor edad se repitieron más CPRE a los mismos pacientes (4-10\%, $\mathrm{p}=0,001)$.

Conclusiones: el éxito y la morbimortalidad para drenar la vía biliar mediante CPRE son similares en los pacientes con edad geriátrica y en los de edad inferior. La coledocolitiasis es más frecuente en los pacientes mayores. No se debe excluir a ningún paciente que precise de una CPRE sólo por la edad.

Palabras clave: CPRE. Geriatría. Obstrucción biliar.

\begin{abstract}
Background and objective: endoscopic retrograde cholangiopancreatography (ERCP) is usually the procedure of choice for relieving bile duct obstruction. A large number of patients undergoing this intervention are geriatric population (aged 75 years of age and older). Our aim was to assess the efficacy of ERCP in this group of patients as compared to younger ones.

Patients and methods: a retrospective study. All patients in whom a therapeutic biliary endoscopy had been performed over a four-year period of time (2002-2005) were included.

Results: 178 geriatric patients and 159 younger ones underwent ERCP. No differences were found in successful biliary drainage (97.7 vs. 98.7\%), complication number (11.8 vs. $14.4 \%)$, or mortality rate (1.1 vs. $0.6 \%)$. On the other hand, more common bile duct stones were found in geriatric patients ( 57.3 vs. $39.6 \%, p=0.004)$, and also more self-expanding metal stents were employed to drain malignant obstructive jaundice (47 vs. $8 \%, p=0.0035)$. In the youngest group, more ERCPs were repeated in the same patients (4 vs. 10\%, p = 0.001).

Conclusions: the geriatric population showed similar success and morbidity \& mortality rates when compared to younger patients in draining their bile duct by means of ERCP. Common bile duct stones were more frequently found in geriatric patients. No patients needing an ERCP should be excluded only because of their age.
\end{abstract}

Key words: ERCP. Geriatrics. Biliary obstruction.

García-Cano J, González Martín JA, Morillas Ariño MJ, Pérez García JI, Redondo Cerezo E, Jimeno Ayllón C, Viñuelas Chicano M, Sánchez Manjavacas N, Gómez Ruiz CJ, Pérez Vigara MG, Pérez Sola A. Resultados del drenaje de la vía biliar por CPRE en pacientes con edad geriátrica. Rev Esp Enferm Dig 2007; 99: 451-456.

Recibido: 29-12-06

Aceptado: 11-05-07

Correspondencia: Jesús García-Cano. C/ Federico Mayor Zaragoza, 2, 5 A. 16002 Cuenca.e-mail: j.garcia-cano@terra.es

\section{INTRODUCCIÓN}

La colangiopancreatografía retrógrada endoscópica (CPRE) es, desde hace más de treinta años, la técnica habitual de elección para desobstruir la vía biliar (1). Los métodos de obtención de imágenes con los que se cuenta en la actualidad, especialmente la ultrasonografía endos- 
cópica y la colangiopancreatografía por resonancia magnética, han convertido a la CPRE en una intervención eminentemente terapéutica.

El carácter mínimamente invasivo de la técnica ha situado a la CPRE en un lugar destacado del intervencionismo endoscópico y apto para aplicarse a una gran variedad de pacientes. Los enfermos con edades avanzadas que precisan una CPRE constituyen una muestra elocuente de las ventajas de esta técnica y del alto índice de beneficio/riesgo que conlleva. De esto, son buen ejemplo las series publicadas, cada vez más frecuentes, de CPRE en pacientes a partir de los 90 años (2).

Por otro lado, en los estudios realizados en pacientes muy ancianos se subraya que esos resultados se han obtenido en centros grandes, con un número elevado de CPRE anuales (más de 200) y que quizás los resultados no sean extrapolables a centros más pequeños (3-5).

A pesar de que los buenos resultados de la CPRE en enfermos mayores de 90 años sean un ejemplo sobresaliente del carácter mínimamente invasivo de la técnica, no dejan de ser un grupo reducido. Por ello, decidimos realizar un estudio sobre los resultados del drenaje biliar endoscópico en pacientes geriátricos, comparando sus resultados con los de menor edad. Teniendo en cuenta, además, que nuestro centro puede considerarse como una unidad pequeña de CPRE.

\section{PACIENTES Y MÉTODOS}

Se ha realizado un estudio retrospectivo de las CPRE con terapéutica biliar realizadas durante un periodo de 4 años (2002-2005). La mayoría de las intervenciones fueron realizadas, en nuestro centro, por dos de los autores (JGC y JGM), con experiencia en el intervencionismo biliar endoscópico.

Durante el procedimiento se empleó sedación consciente con meperidina y midazolam administrada por los mismos endoscopistas. Se administró buscapina para inhibir la motilidad duodenal. En todas las exploraciones, en general asistió el mismo equipo de enfermería y de auxiliares de clínica. Durante la CPRE se monitorizó la frecuencia cardiaca y la saturación sanguínea y se administró a los pacientes oxígeno con cánula nasal. Las CPRE se realizaron en una sala de radiología con posibilidad de fluoroscopia. Los pacientes dieron su consentimiento para la realización de la prueba tras recibir la información médica oportuna.

Se utilizaron duodenoscopios terapéuticos con un canal de trabajo de 4,2 $\mathrm{mm}$. La canulación de la papila de Vater, esfinterotomía, dilatación, inserción de prótesis y otros procedimientos de la CPRE, se realizaron según técnicas previamente descritas (6) (Fig. 1). El fallo terapéutico se definió como la falta de tratamiento endoscópico eficaz, en general un drenaje biliar adecuado. No se consideró fallo terapéutico las coledocolitiasis que no se extrajeron totalmente, si pudo insertarse una prótesis para

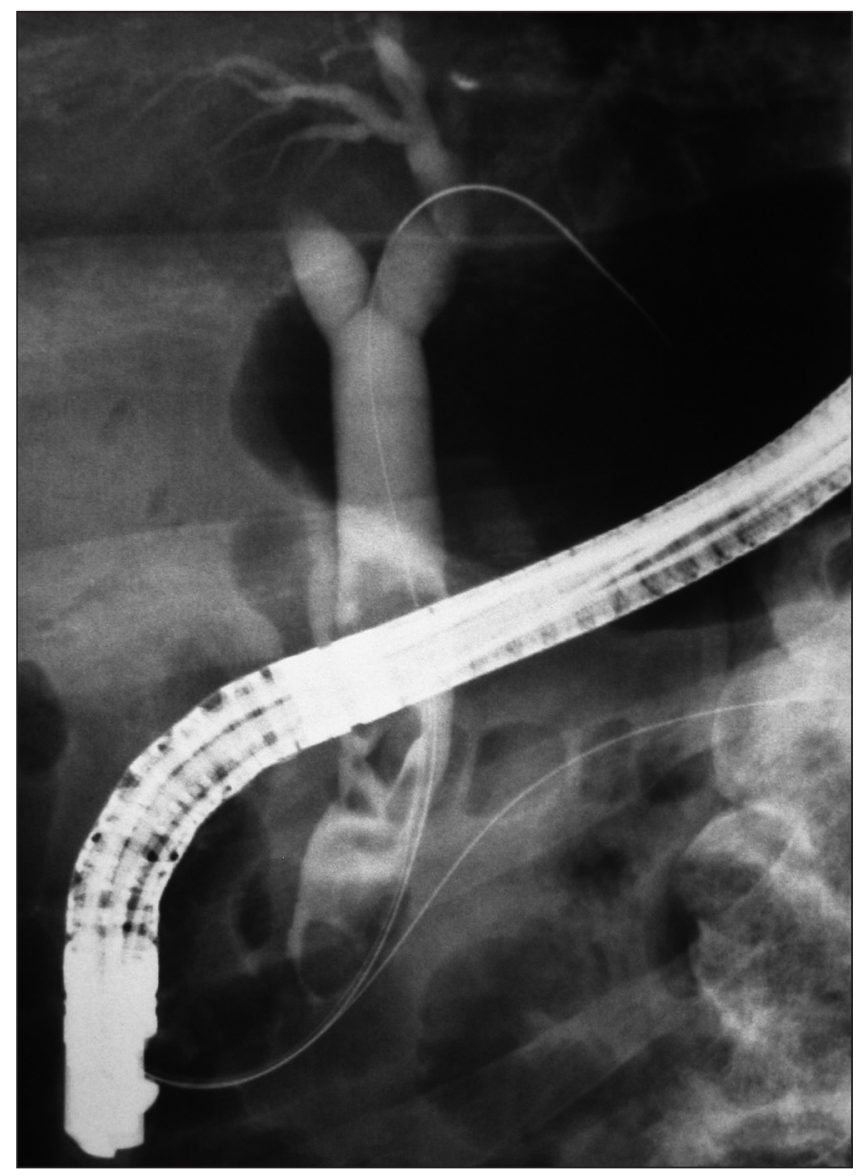

Fig. 1. Paciente con coledocolitiasis. Cuando se canula de forma repetida el páncreas puede dejarse una guía en el Wirsung que sirve como "escalón" para entrar en el colédoco.

mantener un drenaje adecuado (Fig. 2). En general, se procuró insertar una prótesis plástica de 7 french de diámetro (algo más de $2 \mathrm{~mm}$ ) y $9 \mathrm{~cm}$ de longitud, con doble pigtail (arandela). Debido a la forma de este tipo de prótesis es más difícil que migre, tanto dentro del colédoco como al duodeno. Aunque la prótesis pueda obstruirse en algo más de un mes, el flujo de bilis no se produce sólo por dentro, sino también alrededor de ella, especialmente si se ha realizado una esfinterotomía biliar. Además, la prótesis ayuda a impedir la impactación de coledocolitiasis no extraídas en la papila.

Todos los pacientes a los que se realizó la CPRE estuvieron ingresados, al menos, la noche de la intervención. En los días sucesivos se comprobó si presentaban alguna complicación, que se graduaron según un consenso de 1991 (7).

Los datos relativos a la técnica y a las complicaciones se recogieron de forma prospectiva, aunque el análisis global se ha realizado de forma retrospectiva. La comparación de variables cualitativas se hizo mediante la prueba de la Chi cuadrado o la exacta de Fisher, según fuera apropiado. Para las medias se utilizó la t de Student. Se consideró diferencia estadísticamente significativa una $\mathrm{p}<0,05$. 


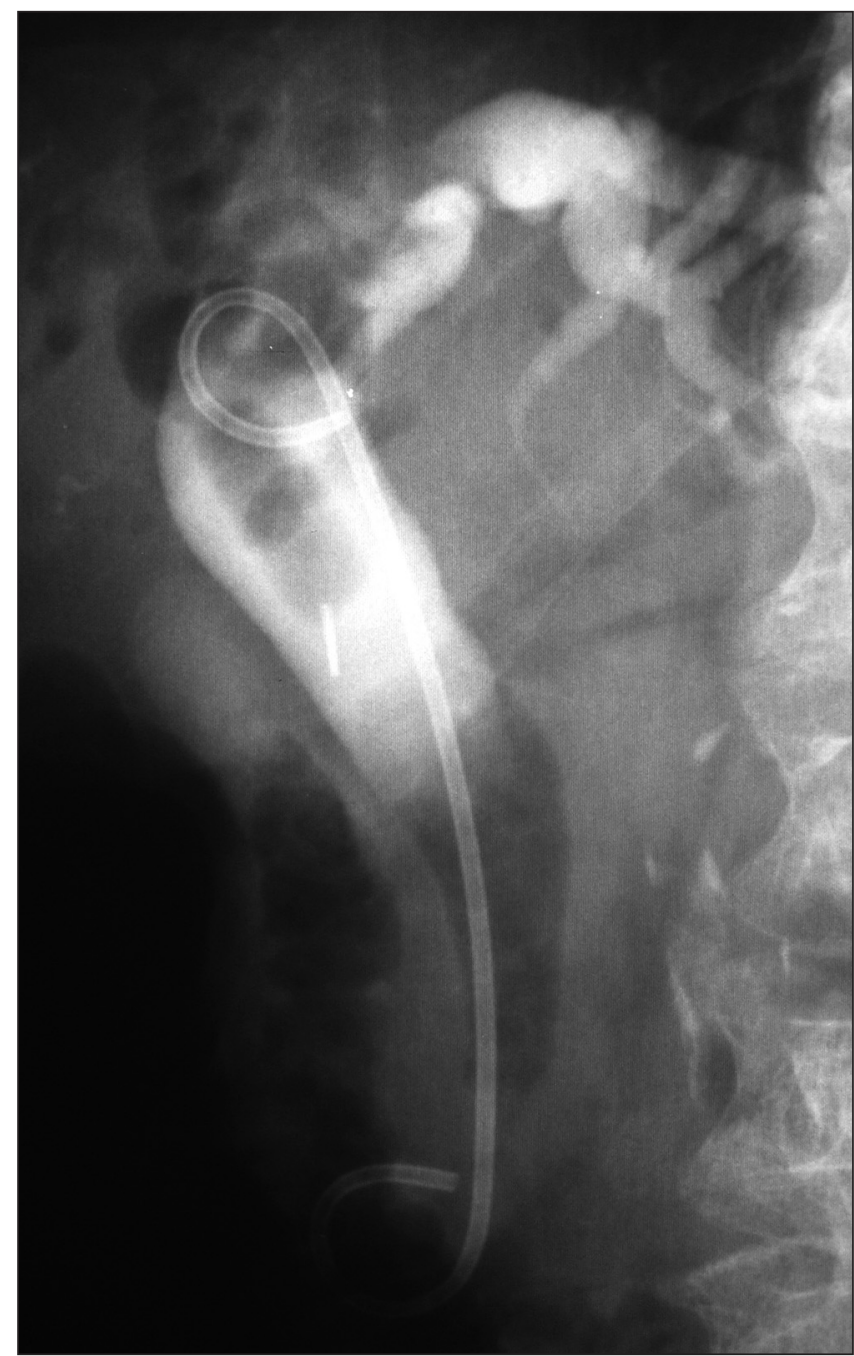

Fig. 2. Prótesis biliar plástica insertada tras no poder extraer completamente una coledocolitiasis de gran tamaño. De esta forma se garantiza el drenaje biliar.

\section{RESULTADOS}

Durante el periodo de estudio se realizó terapéutica biliar endoscópica mediante CPRE a 178 --53\%- pacientes en edad geriátrica (con 75 años o mayores) y a 159 enfermos de menor edad (47\%). Cuando comparamos ambos grupos (Tabla I), encontramos diferencia estadísticamente significativa, lógicamente, en la edad que era un supuesto previo del estudio. Los pacientes geriáticos tenían de media 82 años y los más jóvenes 63 .

Las tasas de éxito para el drenaje biliar fueron similares en ambos grupos (Tabla I), al igual que el porcentaje de complicaciones y la mortalidad. En la tabla II se indican, pormenorizadas, las complicaciones de la CPRE en ambos grupos. En el grupo geriátrico fallecieron dos mujeres (mortalidad 1,1\%). Una de accidente cerebrovascular al finalizar la CPRE y otra por pancreatitis aguda ocasionada por el procedimiento. Entre los pacientes de
Tabla I. Comparación de resultados del drenaje la vía biliar mediante CPRE entre los pacientes con edad geriátrica y los pacientes más jóvenes

\begin{tabular}{lccc}
\hline & $\begin{array}{c}\text { Edad } \\
\text { geriátrica } \\
(n=178)\end{array}$ & $\begin{array}{c}\text { Menores de } \\
75 \text { años } \\
(n=159)\end{array}$ & $\begin{array}{c}\text { Significación } \\
\text { estadistica }\end{array}$ \\
\hline Edad & $82 \pm 5(75-97)$ & $63,6 \pm 10,5(24-74)$ & $p<0,00001$ \\
Hombre/mujer & $87 / 91$ & $85 / 74$ & NS \\
Éxito en el drenaje biliar & $174(97,7 \%)$ & $157(98,7 \%)$ & NS \\
Repetidas & $7(4 \%)$ & $16(10 \%)$ & $p=0,04$ \\
Coledocolitiasis & $102(57,3 \%)$ & $63(39,6 \%)$ & $p=0,001$ \\
Tumores & $38(21,3 \%)$ & $24(15 \%)$ & $N S$ \\
Complicaciones & $21(11,8 \%)$ & $23(14,4 \%)$ & $N S$ \\
Mortalidad & $2(1,1 \%)$ & $1(0,6 \%)$ & NS \\
Duración CPRE & $63 \pm 43$ minutos & $63 \pm 35$ minutos & NS \\
ASA II & $48 / 119(40 \%)$ & $101 / 132(76,5 \%)$ & $p<0,05$ \\
ASA III & $53 / 119(44,5 \%)$ & $29 / 132(22 \%)$ & $p<0,05$ \\
ASA IV & $18 / 119(15 \%)$ & $2 / 132(1,5 \%)$ & $p<0,05$ \\
\hline
\end{tabular}

NS: no significativo; ASA: situación de riesgo anestesiológico basado en la morbilidad de los pacientes.

Tabla II. Complicaciones de la CPRE en ambos grupos

\begin{tabular}{lccc}
\hline & $\begin{array}{c}\text { Edad } \\
\text { Geriátrica }\end{array}$ & $\begin{array}{c}\text { Menores de } \\
75 \text { años }\end{array}$ & $\begin{array}{c}\text { Significación } \\
\text { estadística }\end{array}$ \\
\hline $\begin{array}{l}\text { Pancreatitis } \\
\text { Hemorragia }\end{array}$ & $10(4,7 \%)$ & $11(4,8 \%)$ & NS \\
postesfinterotomía & $7(3,3 \%)$ & $7(3 \%)$ & NS \\
biliar & & & \\
Colangitis & $1(0,4 \%)$ & $1(0,4 \%)$ & NS \\
Perforación & $2(0,9 \%)$ & $1(0,4 \%)$ & NS \\
Otras & $1(0,4 \%)^{*}$ & $3(1,3 \%)^{* *}$ & NS \\
NS: no significativo; *: accidente cerebrovascular; **: edema agudo de pulmón, \\
fiebre (en dos pacientes).
\end{tabular}

menor edad falleció otra paciente por hemorragia tras una esfinterotomía biliar (mortalidad 0,6\%).

Se encontró diferencia estadísticamente significativa en la frecuencia de coledocolitiasis como causante de la obstrucción biliar (Tabla I). La incidencia fue mayor en la edad geriátrica $(57,3 \%)$ que en el grupo de menor edad $(39,6 \%), p=0,004$. Las litiasis coledocianas no pudieron extrarse en la primera sesión endoscópica en 27/102 $(26,4 \%)$ de los pacientes más ancianos y en 12/63 (19\%) ocasiones en los más jóvenes, porcentajes cuyas diferencias no son significativas.

Otra diferencia estadísticamente significativa encontrada es que en los pacientes más jóvenes hubo que repetir más veces la CPRE en los mismos enfermos (4\% en edad geriátrica de procedimientos repetidos frente a $10 \%$ en los de menor edad, $\mathrm{p}=0,001)$. Se debió esto a las dilataciones repetidas, mediante prótesis plásticas, de estenosis benignas de la vía biliar -postquirúrgicas, por pancreatitis crónica- (Fig. 3), que fueron más frecuentes en los pacientes jóvenes, aunque sin alcanzar diferencia significativa con el número de estenosis de la misma naturaleza en los ancianos. Otra de las causas que explican el mayor número de CPRE en el grupo de menor edad son los intentos repetidos para extraer completamente las coledo- 


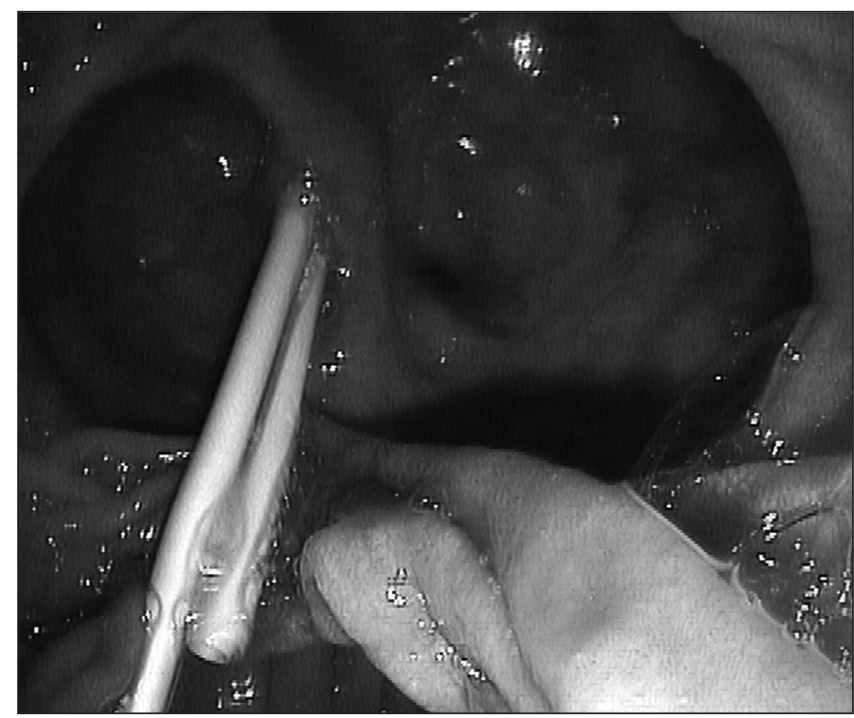

Fig. 3. Papila intradiverticular de la que salen dos prótesis plásticas insertadas para dilatar una estenosis biliar benigna.

colitiasis. En los pacientes en edad geriátrica muchas veces no se repitió la CPRE, sino que la esfinterotomía y la prótesis plástica, que suele insertarse para garantizar el drenaje biliar (Fig. 2), se consideraron un tratamiento a largo plazo. Sin embargo, en los pacientes más jóvenes sí que se realizaron habitualmente más CPRE para intentar limpiar completamente el colédoco.

La frecuencia de ictericia obstructiva neoplásica fue similar en ambos grupos (Tabla I). En el grupo geriátrico hubo 16 tumores de cabeza pancreática, 10 colangiocarcinomas, 3 ampulomas, 7 cánceres de vesícula biliar y dos compresiones de la vía biliar por ganglios metastáticos. Entre los pacientes de menor edad se presentaron 10 cánceres de cabeza de páncreas, 7 colangiocarcinomas, 4 neoplasias de vesícula y dos compresiones por ganglios tumorales. Aunque la frecuencia global fue similar en ambos grupos, sin embargo, en los pacientes en edad geriátrica se utilizaron para descomprimir la vía biliar 18 (47\%) prótesis metálicas autoexpandibles (Fig. 4), mientras que en el grupo de menor edad sólo 2 (8\%), p = 0,0035 . El motivo es que en este grupo se remitieron más pacientes a Cirugía para intentar el tratamiento radical del tumor y se prefirió emplear prótesis biliares plásticas para descomprimir la vía biliar.

La duración media de los procedimientos fue de una hora en ambos grupos.

Dado el carácter retrospectivo del estudio, no pudo determinarse con exactitud en todos los pacientes el estado de riesgo anestesiológico (ASA) establecido según las patologías de base. En el conjunto de enfermos en que pudo realizarse se observó un mayor número de pacientes con riesgo anestesiológico elevado en el grupo geriátrico (Tabla I).

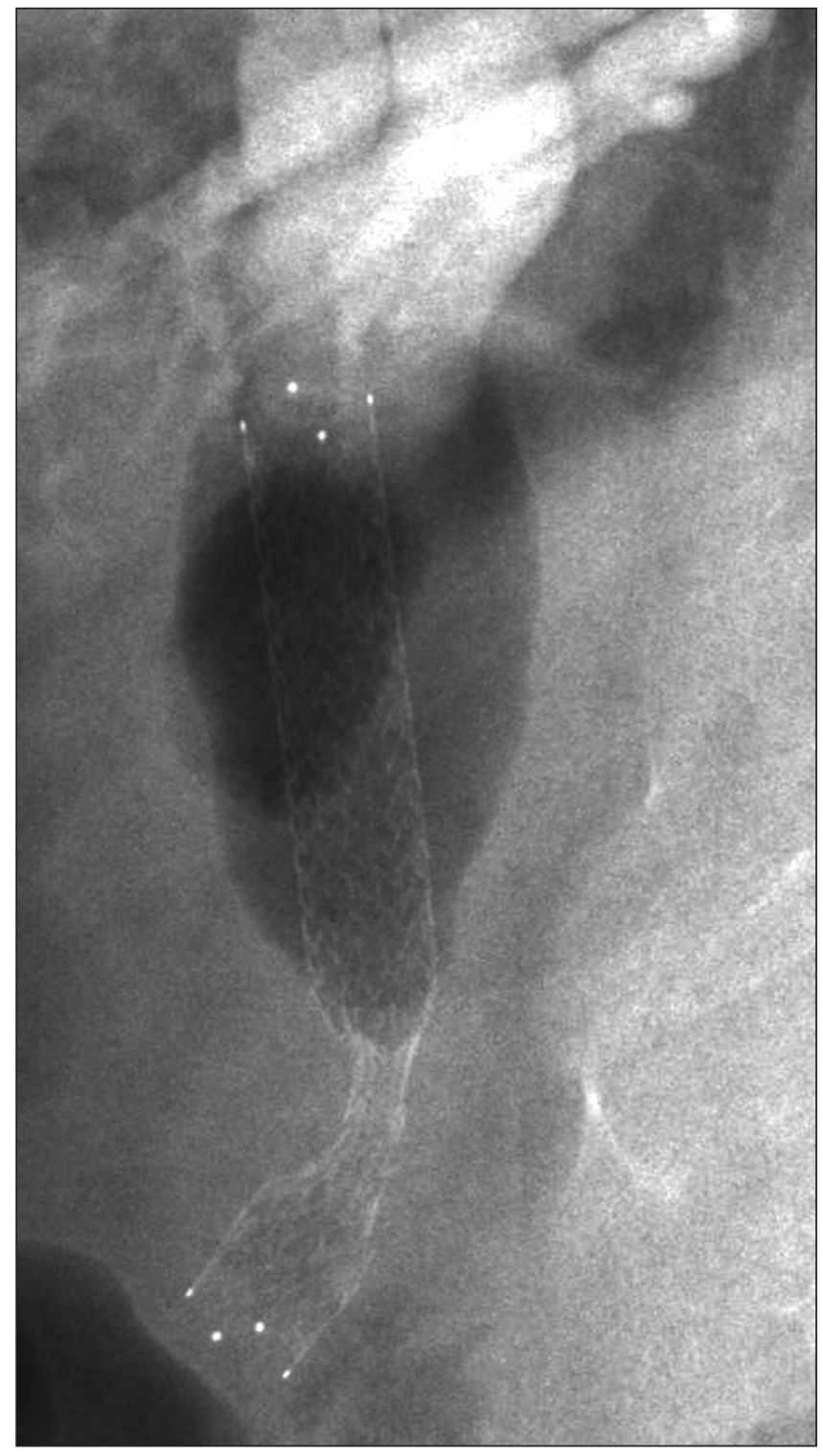

Fig. 4. Paciente de 92 años con ictericia obstructiva por una neoplasia de cabeza de páncreas. Prótesis metálica autoexpandible de nitinol (Zilver) inmediatamente tras su colocación. Se observa la zona de la estenosis en donde la prótesis aún no se ha abierto completamente. La aerobilia dibuja el colédoco muy dilatado.

\section{DISCUSIÓN}

La CPRE es un método consolidado para el tratamiento de la patología de la vía biliar. En 1974 se comunicaron las primeras esfinterotomías biliares endoscópicas para la extracción de cálculos coledocianos $(8,9)$ y en 1979 comenzaron a realizarse los primeros drenajes biliares por medio de prótesis plásticas (10). A pesar de que la CPRE es la técnica más difícil de la endoscopia digestiva, con complicaciones en alrededor del $10 \%$ de los procedimientos y con una mortalidad cercana al $1 \%$ (11), el tiempo ha confirmado y afianzado sus enormes beneficios. 
Cuando sea posible, la CPRE debería realizarse en centros con una dedicación especial y con un número elevado de intervenciones anuales. Sin embargo, la distribución y el acceso de los pacientes a los recursos sanitarios hacen necesario, frecuentemente, realizar esta técnica en hospitales en que se espere un volumen anual reducido (menos de 200 procedimientos al año). En estudios previos de nuestro propio grupo $(12,13)$ y de otros hospitales (14-16) se ha comprobado que la dedicación continua, constante y habitual de pocas personas, en un centro pequeño, puede conseguir unos resultados aceptables en la CPRE cuando sea preciso realizar la técnica. Pensamos que el presente estudio puede ayudar a corroborar que, al igual que en grandes centros, no hay diferencia en los resultados según la edad de los pacientes.

El hecho de poder disponer de una técnica eficaz para drenar la vía biliar con independencia de la edad es muy importante, teniendo en cuenta, por ejemplo, que la incidencia de litiasis afecta a casi un tercio de los pacientes a partir de los 70 años y que la morbimortalidad de la cirugía biliar se incrementa con la edad (17).

En nuestro estudio, la extracción completa de coledocolitiasis en la primera CPRE ha sido similar en ambos grupos. No habíamos obtenido los mismos resultados considerando sólo los pacientes mayores de 90 años (2), en los que parece que los cálculos son más difíciles de extraer (5). En la experiencia de Huguet y cols. (18), estos pacientes mayores de 90 años también presentaban litiasis coledocianas de gran tamaño y de extracción difícil. Cuando tras una CPRE quedan cálculos coledocianos, lo más sencillo es dejar una prótesis plástica para asegurar el drenaje biliar $(19,20)$ (Fig. 2). Posteriormente, pueden realizarse nuevos intentos endoscópicos de extracción o bien considerar la prótesis como un tratamiento parcial definitivo. En nuestros resultados, se observa que el número de CPRE repetidas en los mismos enfermos fue mayor significativamente en los pacientes más jóvenes. Una de las causas fueron los sucesivos procedimientos de extracción de coledocolitiasis realizadas en ellos.

Los pacientes en edad geriátrica presentan más comorbilidad que los más jóvenes (17). Por el carácter retrospectivo de nuestro estudio, sólo ha sido posible cuantificar con precisión el estado anestesiológico (ASA) de los enfermos en un grupo de estos pacientes. Como se observa en la tabla I, el riesgo anestesiológico era mayor en los pacientes geriátricos.

En los pacientes más jóvenes se utilizó un mayor número de prótesis biliares plásticas para paliar la ictericia obstructiva tumoral. El motivo es que se pensó remitir a Cirugía a más de estos pacientes para intentar un tratamiento radical de la enfermedad. Aunque hasta el momento se pensaba que las prótesis metálicas autoexpandibles podían representar una mayor dificultad para efectuar la anastomosis bilio-digestiva en el acto quirúrgico, estudios más recientes señalan que, al menos en es- tenosis distales como las producidas por el cáncer de cabeza de páncreas, no hay más complicaciones quirúrgicas con prótesis metálicas. Además, si la cirugía se retrasa, por ejemplo por radioterapia previa, hay menos riesgo de que la prótesis se obstruya (21).

Como conclusión, en nuestro estudio, el éxito y la morbimortalidad para desobstruir la vía biliar mediante CPRE son similares en pacientes con edad geriátriaca y en enfermos más jóvenes. En los pacientes de más edad existen mayor número de coledocolitiasis y en los más jóvenes se repite más veces la CPRE en los mismos enfermos para tratar estenosis coledocianas benignas y conseguir una limpieza total de las coledocolitiasis. Por lo tanto, ningún paciente que lo necesite, debería ser rechazado para una CPRE exclusivamente por su edad (22).

\section{BIBLIOGRAFÍA}

1. Summerfield JA. Biliary obstruction is best managed by endoscopists. Gut 1988; 29: 741-5.

2. García-Cano J. CPRE en pacientes con 90 o más años: aumenta la evidencia de su seguridad. Gastroenterol Hepatol 2005; 28: 50910

3. Hui CK, Liu CL, Lai KC, et al. Outcome of emergency ERCP for acute cholangitis. In patients 90 years of age and older. Aliment Pharmacol Ther 2004: 19: 1153-8.

4. Rodríguez González FJ, Naranjo Rodríguez A, Mata Tapia L, et al. ERCP in patients 90 years of age and older. Gastrointest Endosc 2003; $58: 220-5$.

5. Katsinelos P, Paroutoglou G, Kountouras J, et al. Efficacy and safety of therapeutic ERCP in patients 90 years of age and older. Gastrointest Endosc 2006; 63: 417-23.

6. García-Cano J, González Martín JA. Bile duct cannulation: Success rates for various ERCP techniques and devices at a single institution. Acta Gastroenterol Belg 2006; 69: 261-7.

7. Cotton PB, Lehman G, Vennes J, Geenen JE, Russell RC, Meyers WC, et al. Endoscopic sphincterotomy complications and their management: An attempt at consensus. Gastrointest Endosc 1991; 37 : 383-93.

8. Classen M, Demling L. Endoskopische Sphinkterotomie der papilla vateri und Steinextraktion aus dem ductus choledochus. Dtsch Med Wochenschr 1974; 99: 496-7.

9. Kawai K, Akasaka Y, Murakami K, Tada M, Kohli Y, Nakajima M. Endoscopic sphincterotomy of the ampulla of Vater. Gastrointest Endosc 1974; 20: 148-51.

10. Soehendra N, Reynders-Frederix V. Palliative gallengang-drainage. Dtsch Med Wochenschr 1979; 104: 206-9.

11. Huibregtese K. Complications of endoscopic sphincterotomy and their prevention (editorial). N Engl J Med 1996; 335: 961-3.

12. García-Cano Lizcano J, González Martín JA, Pérez Sola A, Morillas Ariño J. Tratamiento endoscópico de la ictericia obstructiva en un hospital de nivel II del Sistema Nacional de Salud. Gastroenterol Hepatol 2001; 24: 287-91.

13. García-Cano Lizcano J, González Martín JA, Morillas Ariño J, Pérez Sola A. Complications of endoscopic retrograde cholangiopancreatography. A study in a small ERCP unit. Rev Esp Enferm Dig 2004; 96: 163-73.

14. Schlup MMT, Williams SM, Barbezat GO. ERCP: a review of technical competency and workload in a small unit. Gastrointest Endosc 1997; 46: 48-52.

15. Yarze JC, Herlihy KJ, Chase MP, Fritz HP. ERCP experience in a community-based, private-practice setting. Am J Gastroenterol 2000; 95: 3006-7.

16. Ofuafur K, Mehta TA, Roberts K, Sweis R, Parker DR. Safety of Endoscopic Retrograde Cholangio-Pancreatography (ERCP) in a 
small center. Gastrointest Endosc 2005; 61: AB120.

17. Fritz E, Kirchgatterer A, Hubner D, et al. ERCP is safe and effective in patients 80 years of age and older compared with younger patients. Gastrointest Endosc 2006: 899-905

18. Huguet JM, Sempere J, Bort I, Caselles P, Rodríguez E, Durá AB, et al. Complicaciones de la colangiopancreatografía retrógrada endoscópica en pacientes mayores de 90 años. Gastroenterol Hepatol 2005; $28: 263-6$

19. García-Cano Lizcano J, González Martín JA, Pérez Sola A, et al Success rate of complete extraction of common bile duct stones at first endoscopy attempt. Rev Esp Enferm Dig 2002; 94: 346-50
20. García-Cano Lizcano J, González Martín JA Taberna Arana L, Racionero M, Morillas Ariño MJ, Pérez Sola A. Prótesis biliares plásticas en cálculos coledocianos no extraíbles endoscópicamente. Revista de la Asociación Castellana de Aparato Digestivo (ACAD) 2003; 19: 3-6

21. Wasan SM, Ross WA, Saerckel GA, Lee JH. Use of expandable metallic biliary stents in resectable pancreatic cancer. Am J Gastroenterol 2005; 100: 2056-61

22. García-Cano J, Taberna-Arana L. Advanced age per se should not be a contraindication for ERCP intervention. Gastrointest Endosc 2006; 64: 296-7. 